\title{
Monte Carlo Simulations of Cosmic Ray Hadronic Interactions
}

\author{
E Aguayo \\ JL Orrell \\ RT Kouzes
}

April 2011

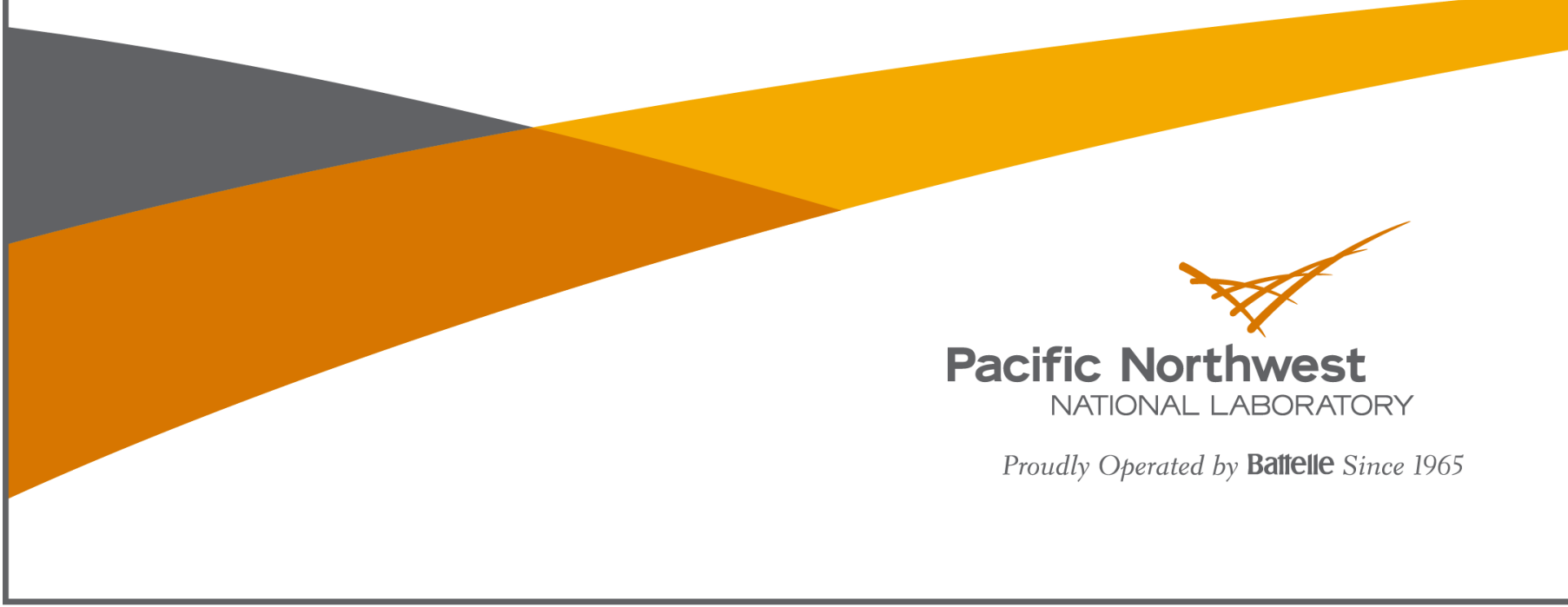




\title{
DISCLAIMER
}

This report was prepared as an account of work sponsored by an agency of the United States Government. Neither the United States Government nor any agency thereof, nor Battelle Memorial Institute, nor any of their employees, makes any warranty, express or implied, or assumes any legal liability or responsibility for the accuracy, completeness, or usefulness of any information, apparatus, product, or process disclosed, or represents that its use would not infringe privately owned rights. Reference herein to any specific commercial product, process, or service by trade name, trademark, manufacturer, or otherwise does not necessarily constitute or imply its endorsement, recommendation, or favoring by the United States Government or any agency thereof, or Battelle Memorial Institute. The views and opinions of authors expressed herein do not necessarily state or reflect those of the United States Government or any agency thereof.

\author{
PACIFIC NORTHWEST NATIONAL LABORATORY \\ operated by \\ BATTELLE \\ for the \\ UNITED STATES DEPARTMENT OF ENERGY \\ under Contract DE-AC05-76RL01830
}

Printed in the United States of America

$$
\begin{aligned}
& \text { Available to DOE and DOE contractors from the } \\
& \text { Office of Scientific and Technical Information, } \\
& \text { P.O. Box 62, Oak Ridge, TN 37831-0062; } \\
& \text { ph: (865) 576-8401 } \\
& \text { fax: (865) 576-5728 } \\
& \text { email: reports@adonis.osti.gov }
\end{aligned}
$$

Available to the public from the National Technical Information Service

5301 Shawnee Rd., Alexandria, VA 22312 ph: (800) 553-NTIS (6847)

email: orders@ntis.gov <http://www.ntis.gov/about/form.aspx>

Online ordering: http://www.ntis.gov 


\title{
Monte Carlo Simulations of Cosmic Ray Hadronic Interactions
}

\author{
E Aguayo \\ RT Kouzes \\ JL Orrell
}

April 2011

Prepared for

the U.S. Department of Energy

under Contract DE-AC05-76RL01830

Pacific Northwest National Laboratory

Richland, Washington 99352 



\section{Summary}

This document describes the construction of and results from the Majorana Cosmic Ray (MaCoR) software tool, developed to model the hadronic interactions of cosmic rays with different geometries and materials. The ubiquity of cosmic radiation in the environment results in the activation of stable isotopes, referred to as cosmogenic activation. The objective is to use this application in conjunction with a model of the MAJORANA DEMONSTRATOR components, from extraction to deployment, to evaluate cosmogenic activation of such components before deployment. The cosmic ray showers include several types of particles with a wide range of energy ( $\mathrm{MeV}$ to $\mathrm{GeV}$ ). It is infeasible to compute an exact result with a deterministic algorithm for this problem; Monte Carlo simulations are a more suitable approach to model cosmic ray hadronic interactions. The tool is based on the Geant 4 toolkit. This toolkit was chosen for its end to end nature and ability to simulate energies up to $\mathrm{GeV}$. Another aspect of Geant 4 that was used in this work is its flexibility and ability to accommodate external source particles libraries. Other tools such as MCNP or FLUKA lack part of these features. In order to validate the results generated by the application, a test comparing experimental muon flux measurements and those predicted by the application is presented. The experimental and simulated results have a deviation of $3 \%$. 



\section{Acronyms and Abbreviations}

$\begin{array}{ll}\text { Ov } \beta \beta & \text { Neutrino-less Double Beta Decay } \\ \text { CERN } & \text { European Center for Nuclear Research } \\ \text { CRY } & \text { Proton-induced Cosmic RaY cascades in the Atmosphere } \\ \text { G4NDL } & \text { Geant4 Nuclear Data Library } \\ \text { Geant4 } & \text { GEometry ANd Transport } 4 \\ \text { GERDA } & \text { GERmanium Detector Array } \\ \text { HPGe } & \text { High Purity Germanium } \\ \text { INFN } & \text { Italian National Institute for Nuclear Physics } \\ \text { LLNL } & \text { Lawrence Livermore National Laboratory } \\ \text { MJD } & \text { MAJORANA DEMONSTRATOR } \\ \text { MaCoR } & \text { Majorana COsmic Ray simulation tool } \\ \text { PNNL } & \text { Pacific Northwest National Laboratory }\end{array}$





\section{Contents}

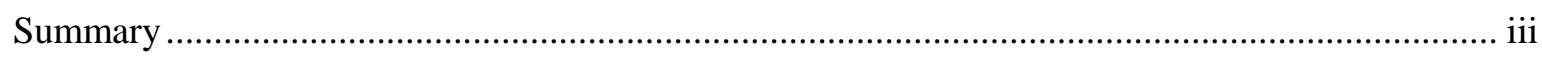

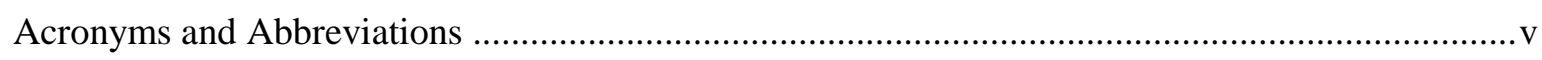

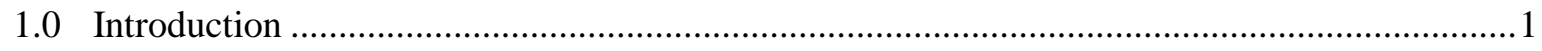

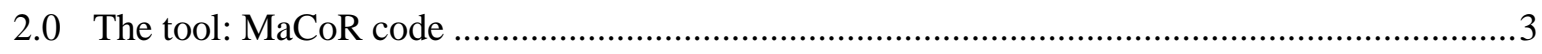

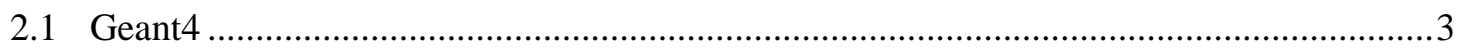

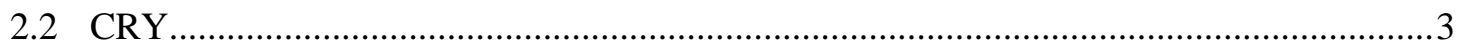

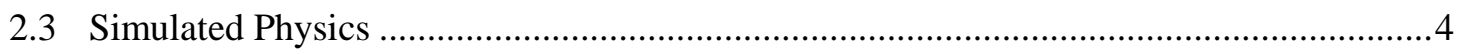

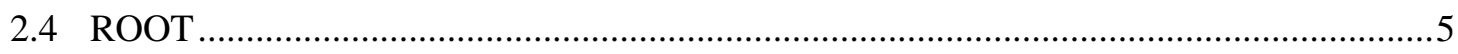

3.0 Results of the simulation of sea level cosmic showers interacting with different materials ......5

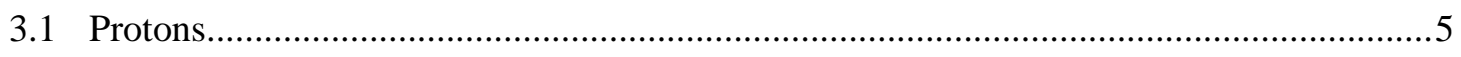

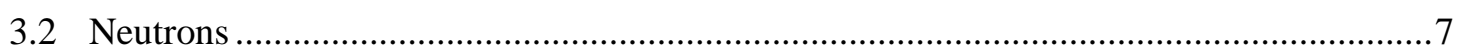

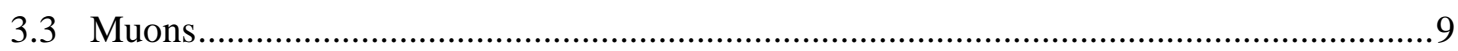

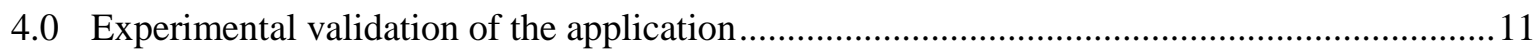

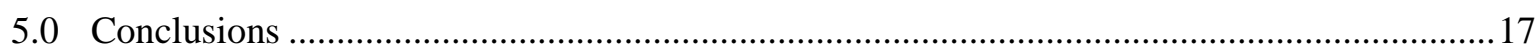

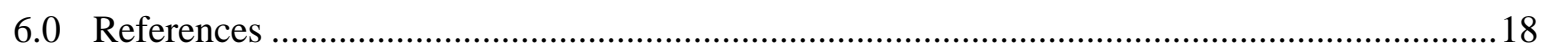




\section{Figures}

Figure 1: Simulation Results for Sea Level Protons Through $10 \mathrm{~cm}$ of Material...........................6

Figure 2: Simulation Results for Sea Level Neutrons Through $10 \mathrm{~cm}$ of Material. ........................8

Figure 3: Simulation Results for Sea Level Muons Through $10 \mathrm{~cm}$ of Material............................ 10

Figure 4: Muon Attenuation as a Function of Dirt Depth........................................................11

Figure 5: Simulation Result of the PNNL Shallow Lab. ............................................................ 12

Figure 6: Simulated Muon Flux Mean Energy as a Function of Depth ....................................... 13

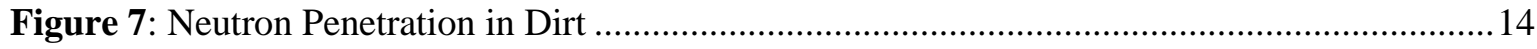

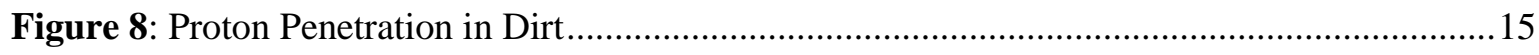

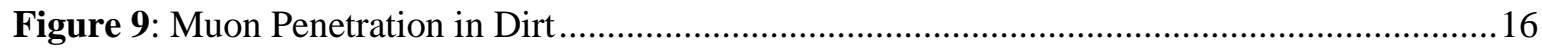

\section{Tables}

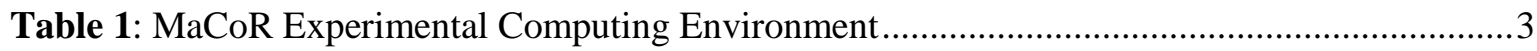

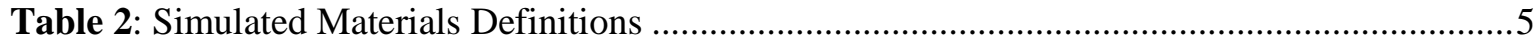

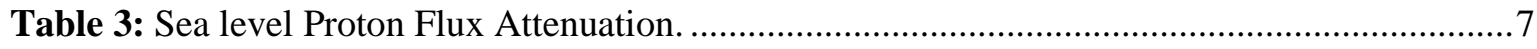

Table 4: Sea level Neutron Flux Attenuation......................................................................... 9

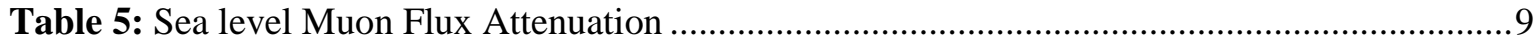




\subsection{Introduction}

The MAJORANA DEMONSTRATOR (MJD) is an ultra-low-background experiment that has been designed to operate with up to $40 \mathrm{~kg}$ of HPGe detectors. The purpose of this experiment is to look for an extraordinarily rare nuclear decay process, $0 v \beta \beta$ [Aalseth 2010]. If observed, the Majorana nature of the neutrino could be determined and thus demonstrate violation of lepton number conservation. The $0 v \beta \beta$ reaction channel in Ge has a decay energy $2039 \mathrm{keV}$. The half-life of such a process is $\sim 10^{-26}$ years. Any other effect depositing energy in the signal region is a background to the $0 v \beta \beta$ signal of interest. Cosmic radiation is bombarding Earth constantly. Exposure time and intensity of cosmic rays striking the experiment's components must be accounted for during fabrication and deployment. Cosmic ray particles undergoing interactions with materials on Earth can produce radionuclides. Among these, there are some interactions that can result in the production of isotopes that deposit energy in the $0 v \beta \beta$ region of interest. In particular, two have been identified as potentially the most important radionuclides, ${ }^{68} \mathrm{Ge}$ and ${ }^{60} \mathrm{Co}$, which can deposit energy in the signal region of the $0 v \beta \beta$ energy spectrum. The cosmic rays impinging an unshielded detector above ground (several per second) will prohibit observing any rare event due to the cosmogenic activity produced in the detector. Underground, only a fraction of the hard component of the cosmic rays (mostly muons) survive.

An idea of how many interfering isotopes are present in the experiment is mandatory in order to validate the results drawn from the data. Ideally, you could $\log$ the exposure time and the intensity of cosmic rays measured in situ at every step of the experiment materials manufacturing process. The tracking must be continuous from raw material through manufacture to deployment. Once the experiment is deployed a sum of the product of exposure intensity and production rate, integrated in time would give an estimate of the anticipated decay rates from cosmogenic isotopes that are present inside the experiment's shield. This procedure is not feasible. The parts will move between various countries, personnel and facilities. Furthermore every source of raw material used to build MJD parts should be considered in the cosmogenic production rate calculation. This document presents an application that models cosmic ray exposure that could ease the complexity of this task. This model could characterize every environment where the parts are stored or worked on, avoiding the need to make measurements in every location, and just record the altitude, shield material and thickness of every environment that the MJD parts see before deployment. Nonetheless, despite the creation of this model, critical parts of the experiment, such as the detector raw enriched germanium, are planned to be followed with cosmic ray detectors until deployment.

The cosmogenic production of a radionuclide is a compound process involving different nuclear particles present in cosmic ray showers and the composition of the materials with which they interact. The wide range of energies of these particles makes experimental measurement of the production rate a difficult task. With this very broad energy range, the physics processes that could produce the isotopes of interest make the complexity of such calculations unapproachable analytically. Monte Carlo simulations have the potential to be used to estimate these compound probabilities. This document describes a tool based on Monte Carlo techniques for modeling of hadronic interactions and cosmic rays. The Majorana Cosmic Ray simulation tool (MaCoR) is presented in Section 2. The code structure description includes the versions of the libraries and environment where the tool was developed. Section 3 is composed of simulation results of different materials, analyzed per particle component of the cosmic ray showers. The code is validated by a comparison of data from an experimental result and analytical descriptions of muon attenuation versus depth. These results are presented in Section 4. 



\subsection{The tool: MaCoR code}

The MaCoR code is built using several nuclear physics toolkits. There are three major components of the application. MaCoR extensively uses the Geant4 [Agostinelli 2003] toolkit to model the physics and transport of the simulated particles. The cosmic shower modeling in MaCoR is based on the CRY library [Hagmann 2011]. MaCoR uses functions with ROOT [ROOT 2011] to output data. The application is written entirely in $\mathrm{C}++$, and the results presented in this document were produced by the application under the environment described in Table 1.

\begin{tabular}{|ccc|}
\hline Parameter & Value & Version \\
\hline Processor & Intel T2400 & $2 @ 1.83 \mathrm{GHz}$ \\
\hline Operating system & Linux & i686 2.6.35 \\
\hline C++ compiler & gcc & 4.5 .1 \\
\hline
\end{tabular}

Table 1: MaCoR Experimental Computing Environment

\subsection{Geant4}

Geant4 is a powerful toolkit for the simulation of the passage of particles through matter. The Geant4 reference paper published in Nuclear Instruments and Methods A in 2003 has become the most cited publication in the whole Nuclear Science and Technology category of Thomson-Reuter's Journal Citation Reports. It is currently the second most cited article among the publications authored by two major research institutes, CERN and INFN [Pia 2009]. The version of Geant4 used to build MaCoR is 4.9.3.p01. The choice to use Geant4 versus a popular Monte- Carlo code such as MCNPX is the ability of Geant to reach energies up to GeV. Other shielding studies using MCNPX study attenuation up to $20 \mathrm{MeV}$ [Stewart 2006]. Geant4 is also capable of simulating physics for protons, muons and neutrons among others. Codes such as SHIELD, only take into account activation from neutrons and do not consider the secondary production from muons and protons in its results [Baravanov 2006].

\subsection{CRY}

The CRY library is used in MaCoR to generate correlated cosmic-ray particle showers at one of three elevations $(0 \mathrm{~m}, 2100 \mathrm{~m}$, and $11300 \mathrm{~m})$ for use as input to transport and detector simulation codes. In the case of MaCoR, Geant 4 is the transport code. CRY provides all particle production (muons, neutrons, protons, electrons, photons, and pions) within a specified area (up to $300 \mathrm{~m}$ by $300 \mathrm{~m}$ ) as well as time of arrival and zenith angle of secondary particles. The library also provides basic correlations between particles within the shower, latitude, and solar cycle variations. During the development of the MaCoR application a new CRY version was released by its creators at LLNL. The result presented in section 3 and 4 of this document were computed using CRY v1.5. The new version, CRY v1.6 was later incorporated into MaCoR. The new version addresses, among other issues, a bug concerning a non-flat 
XY spatial distribution of the generated cosmic rays. Comparison between results of the two versions of the library were performed when CRY v1.6 was released and the new release is consistent with results from MaCoR using the previous version. For all the data presented in this document the CRY library was used in MaCoR with the following parameters: altitude: $0 \mathrm{~m}$, date: 01/15/2011, latitude: 45 degrees and a simulated surface area of $1 \mathrm{~m} \times 1 \mathrm{~m}$.

\subsection{Simulated Physics}

The physics list in MaCoR has been optimized for the reliable simulation of the signal process and the most common background sources in double beta-decay, according to the suggestions of the Geant 4 team. Relevant physics for the application are those that contribute to one of the effects listed below;

- All nuclear reactions involving the particles of interest (neutron, proton and muon)

- ${ }^{68} \mathrm{Ge}$ and ${ }^{60} \mathrm{Co}$ isotope production

The MaCoR physics list is based on the underground physics advanced example which is distributed with Geant 4 . The hadronic models implemented in the physics list are:

- Theory-driven quark-gluon string models (QGSP v2.4) for pions, kaons and nuclei with energies up to $100 \mathrm{TeV}$.

- Bertini cascade models describing nucleon and pion interactions below energies of $10 \mathrm{GeV}$. See text below

- Tabulated cross-section data derived from the ENDF/B-VI database [NNDC 2011] to model capture, fission, elastic scattering and inelastic scattering of neutrons with energies from thermal up to energies of $20 \mathrm{MeV}$ (high precision models). This is possible thanks to the way Geant 4 can use different cross-sections independent from the way they are accessed. The specific version of this model is G4NDL3.14

- Interactions of high-energy muons are simulated using the G4MuNuclearInteraction model [Bogdanov 2006]. This model includes the following processes: ionization by high energy muons with radiative corrections, electron-positron pair production, muon induced nuclear reactions, gamma annihilation into muon pair, and into pion pair.

The Bertini cascades model generates the final state for hadron inelastic scattering by simulating the intra-nuclear cascade. The model reproduces incident hadrons colliding with protons and neutrons in the target nucleus and produces secondaries, which in turn collide with other nucleons. The final state of each collision is sampled according to free-particle cross section data. The target nucleus is treated as an average nuclear medium to which excitons (particle-hole states) are added after each collision. At the end of the cascade, the excited nucleus is represented as a sum of particle-hole states, which is then decayed by pre-equilibrium, nucleus explosion, fission and evaporation methods. This model reproduces detailed cross section data for nucleons, pions and kaons in the region below $1 \mathrm{GeV}$ and is expected to do reasonably well in the multi-GeV region [GEANT 2011]. 


\subsection{ROOT}

ROOT is an object-oriented library developed by CERN. It was originally designed for particle physics data analysis. MaCoR uses this library to generate the histograms presented in this document. The version of the library used in the generation of the graphs presented in this document was 5.28.

\subsection{Results of the simulation of sea level cosmic showers interacting with different materials}

A straightforward application for the MaCoR tool is to characterize the behavior of materials when exposed to cosmic rays. In this section the three particle components most interesting for cosmogenic activation studies (protons, neutrons and muons) are analyzed separately. This is done using the CRY feature that lets the user choose the particles of interest from the cosmic ray shower. The analysis presented involves the study of four different materials, chosen for their applicability to cosmic shield design. These materials are dirt, iron, lead and polyethylene. Table 2 contains the list of properties of these material used in the simulation. The composition of dirt is that reported in [BIO 2011]. In all the simulations presented in this section the thickness of the material in the simulated geometry is $10 \mathrm{~cm}$ and the particle source surface area is determined by the CRY library and is set to $1 \mathrm{~m} \mathrm{x} 1 \mathrm{~m}$ in the results presented in this section. All simulation executions were bounded to $10^{5}$ events. The results presented in this section were normalized in time.

\begin{tabular}{cccc}
\hline Material & Density $(\mathrm{g} / \mathrm{cm} 3)$ & Formulation & $\begin{array}{c}\text { Element composition } \\
(\%)\end{array}$ \\
\hline Dirt & 1.52 & $(\mathrm{C}+\mathrm{Si}+\mathrm{Al}+\mathrm{Fe}+\mathrm{O})$ & Carbon 6 $(1 \%)$ \\
& & Silicon $14(29 \%)$ \\
& & Aluminum $13(15 \%)$ \\
& & Iron $26(5 \%)$ \\
Iron & 7.87 & & Oxygen $8(50 \%)$ \\
Lead & 11.35 & $\mathrm{Fe}$ & Iron $26(100 \%)$ \\
Poly-ethylene & 0.94 & $\mathrm{~Pb}$ & Lead $86(100 \%)$ \\
& & $\mathrm{C}_{2} \mathrm{H}_{4}$ & Carbon $6(85 \%)$ \\
& & Hydrogen $1(15 \%)$ \\
\hline
\end{tabular}

Table 2: Simulated Materials Definitions

\subsection{Protons}

The proton component of the cosmic ray shower is the most active in terms of secondary generation of the three particles considered in this section. This means that an effective shield against protons will induce large numbers of secondary neutrons. The most prominent result is that the traditional material for shield construction, lead, is where the greatest amount of low energy neutrons are created in comparison with the other three materials. Figure 1 shows the simulated spectra for all four materials (from top left 
clockwise: dirt, iron, polyethylene, lead). The attenuation of protons is tightly correlated with the atomic number of the material.
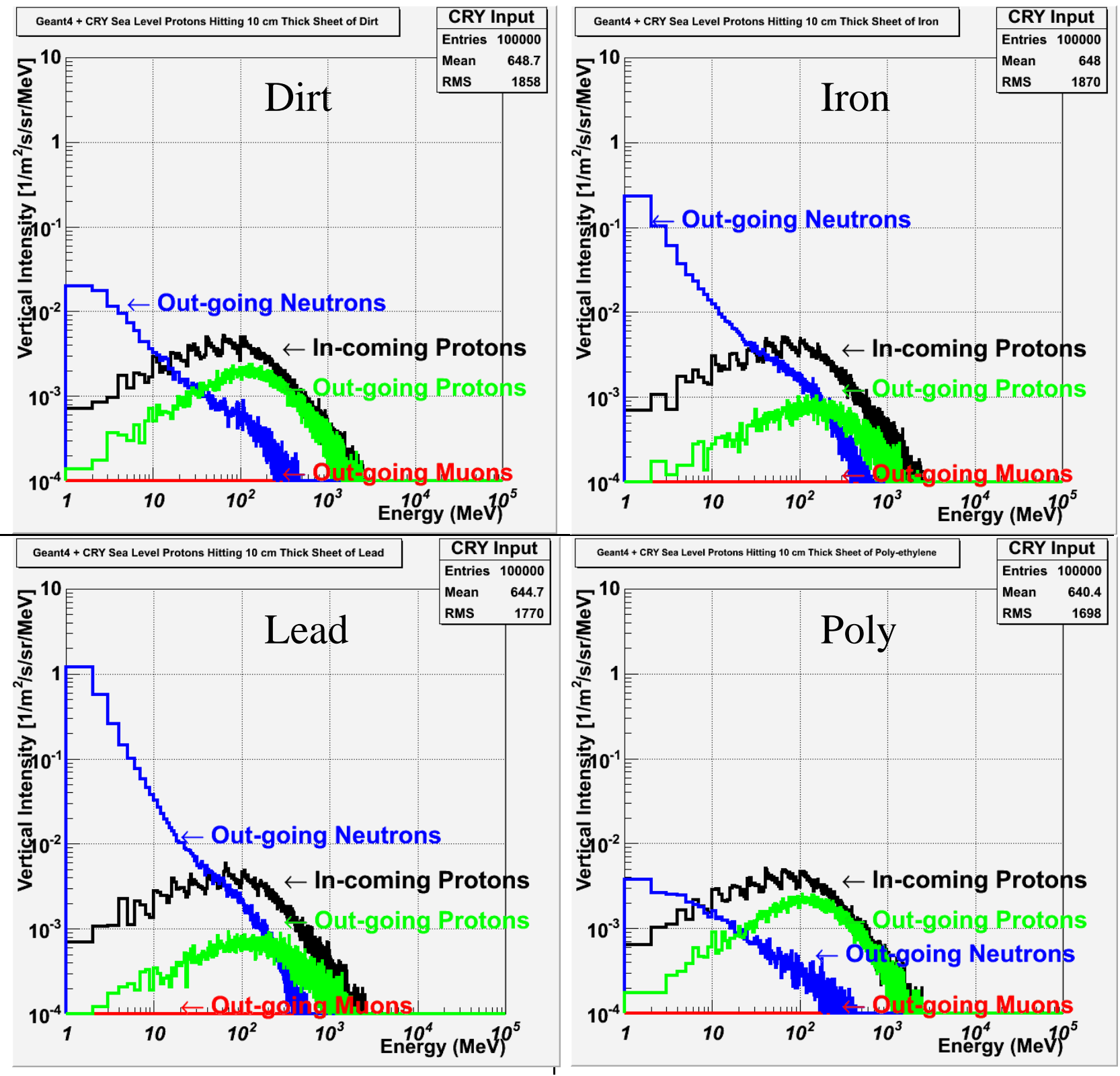

Figure 1: Simulation Results for Sea Level Protons Through $10 \mathrm{~cm}$ of Material.

Table 3 presents the proton attenuation factors for the four materials considered. This value is computed as $100 \%$ minus the ratio of in-coming proton number and out-going proton number. The fraction of generated secondary muons and neutrons are calculated in this table as the ratio of in-coming protons over out-going proton, out-going muons and neutrons. 
Table 3: Sea level Proton Flux Attenuation.

\begin{tabular}{cccc}
\hline Material & $\begin{array}{c}\text { Proton attenuation } \\
(\%)\end{array}$ & $\begin{array}{c}\text { Neutron } \\
\text { generation }(\%)\end{array}$ & $\begin{array}{c}\text { Muon generation } \\
(\%)\end{array}$ \\
\hline Dirt & 45 & 18 & $9 \mathrm{e}-5$ \\
Iron & 68 & 110 & $6 \mathrm{e}-5$ \\
Lead & 71 & 326 & $8 \mathrm{e}-5$ \\
Poly & 30 & 9 & $1 \mathrm{e}-4$ \\
\hline
\end{tabular}

\subsection{Neutrons}

In contrast to proton behavior, energetic neutrons are harder to shield against and their secondary generation is not as prominent. Figure 2 shows the simulated spectra for all four materials (from top left clockwise: dirt, iron, polyethylene, lead). The attenuation of neutrons is not correlated with the atomic number of the material, but correlated with the amount of hydrogen atoms that interact with low energy neutrons, as is the case of polyethylene. The other materials studied only moderate neutrons rather that attenuate them. 


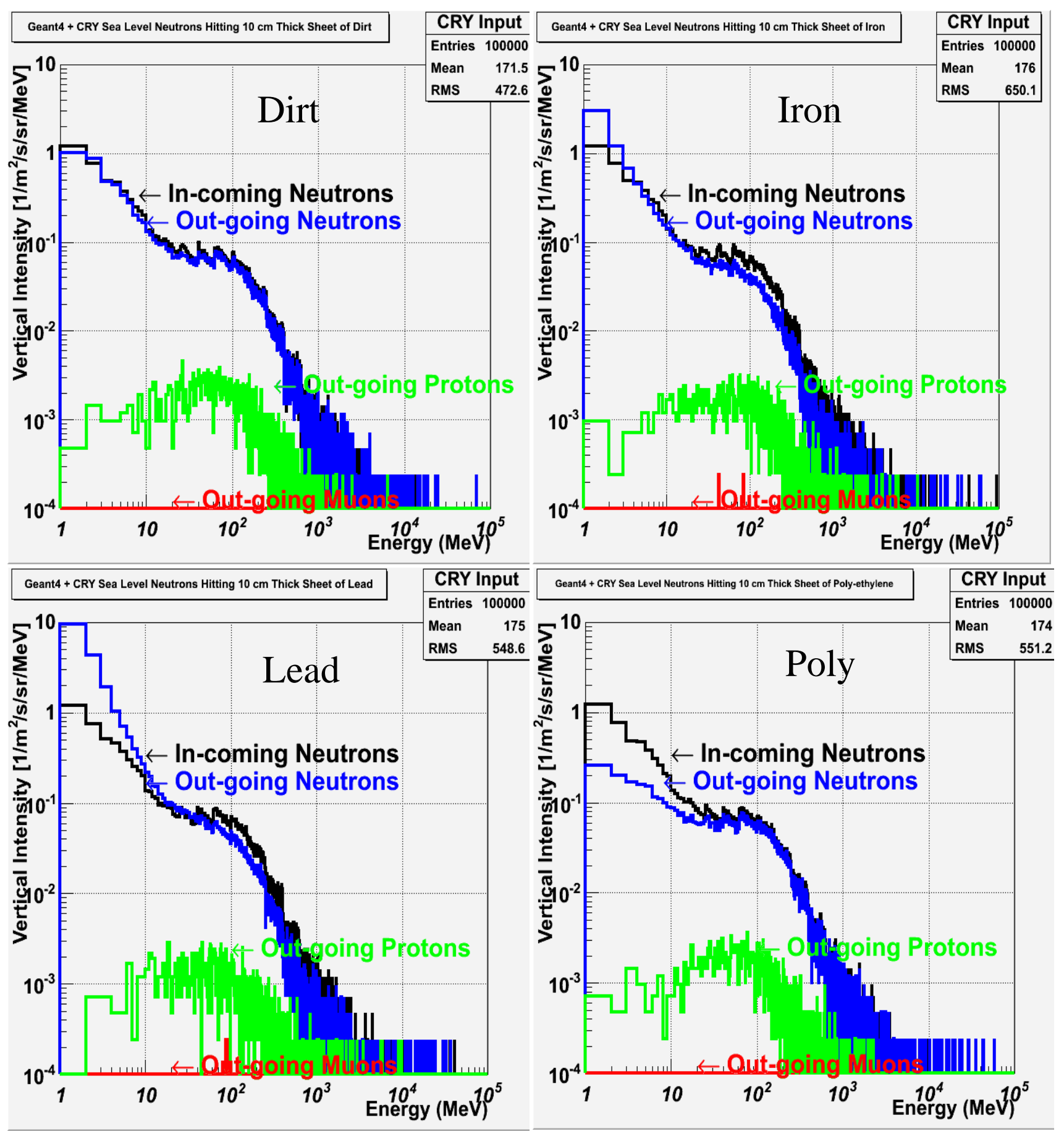

Figure 2: Simulation Results for Sea Level Neutrons Through $10 \mathrm{~cm}$ of Material.

Table 4 presents the neutron attenuation factors for the four materials considered. This value is computed as $100 \%$ minus the ratio of in-coming neutrons to out-going neutrons. The fraction of generated secondary muons and protons are calculated in this table as the ratio of in-coming neutrons over out-going neutrons, out-going muons and protons. A negative attenuation should be read as generated neutrons, instead of attenuated. 
Table 4: Sea level Neutron Flux Attenuation.

\begin{tabular}{cccc}
\hline Material & $\begin{array}{c}\text { Neutron } \\
\text { attenuation }(\%)\end{array}$ & $\begin{array}{c}\text { Proton generation } \\
(\%)\end{array}$ & $\begin{array}{c}\text { Muon generation } \\
(\%)\end{array}$ \\
\hline Dirt & 14 & 2 & $6 \mathrm{e}-5$ \\
Iron & -18 & 2 & $3 \mathrm{e}-5$ \\
Lead & -114 & 2 & $2 \mathrm{e}-5$ \\
Poly & 30 & 2 & $1 \mathrm{e}-5$ \\
\hline
\end{tabular}

\subsection{Muons}

As expected, the muon component of the cosmic ray shower is the hardest to attenuate. Figure 3 shows the simulated spectra for all four materials (from top left clockwise: dirt, iron, polyethylene, lead). The attenuation of muons with this small amount of material is not very informative. The next section of this document presents a more detailed study of the muon component of the cosmic rays. The only noticeable effect is the creation of secondary neutrons. The amount generated has a material density correlation. Polyethylene, the least dense material of those evaluated, is the one that generates the fewest secondary neutrons.

Table 5 presents the muon attenuation factors for the four materials considered. This value is computed as $100 \%$ minus the ratio of out-going muons to in-coming muons. The fraction of generated secondary neutrons and protons are calculated in this table as the ratio of in-coming muons over out-going muons, out-going neutrons and protons, respectively.

Table 5: Sea level Muon Flux Attenuation

\begin{tabular}{cccc}
\hline Material & $\begin{array}{c}\text { Muon attenuation } \\
(\%)\end{array}$ & $\begin{array}{c}\text { Proton generation } \\
(\%)\end{array}$ & $\begin{array}{c}\text { Neutron } \\
\text { generation }(\%)\end{array}$ \\
\hline Dirt & 0.5 & 0 & $7 \mathrm{e}-4$ \\
Iron & 2.8 & 0 & $8 \mathrm{e}-3$ \\
Lead & 3.4 & 0 & 1.1 \\
Poly & 0.4 & 0 & $2 \mathrm{e}-5$ \\
\hline
\end{tabular}




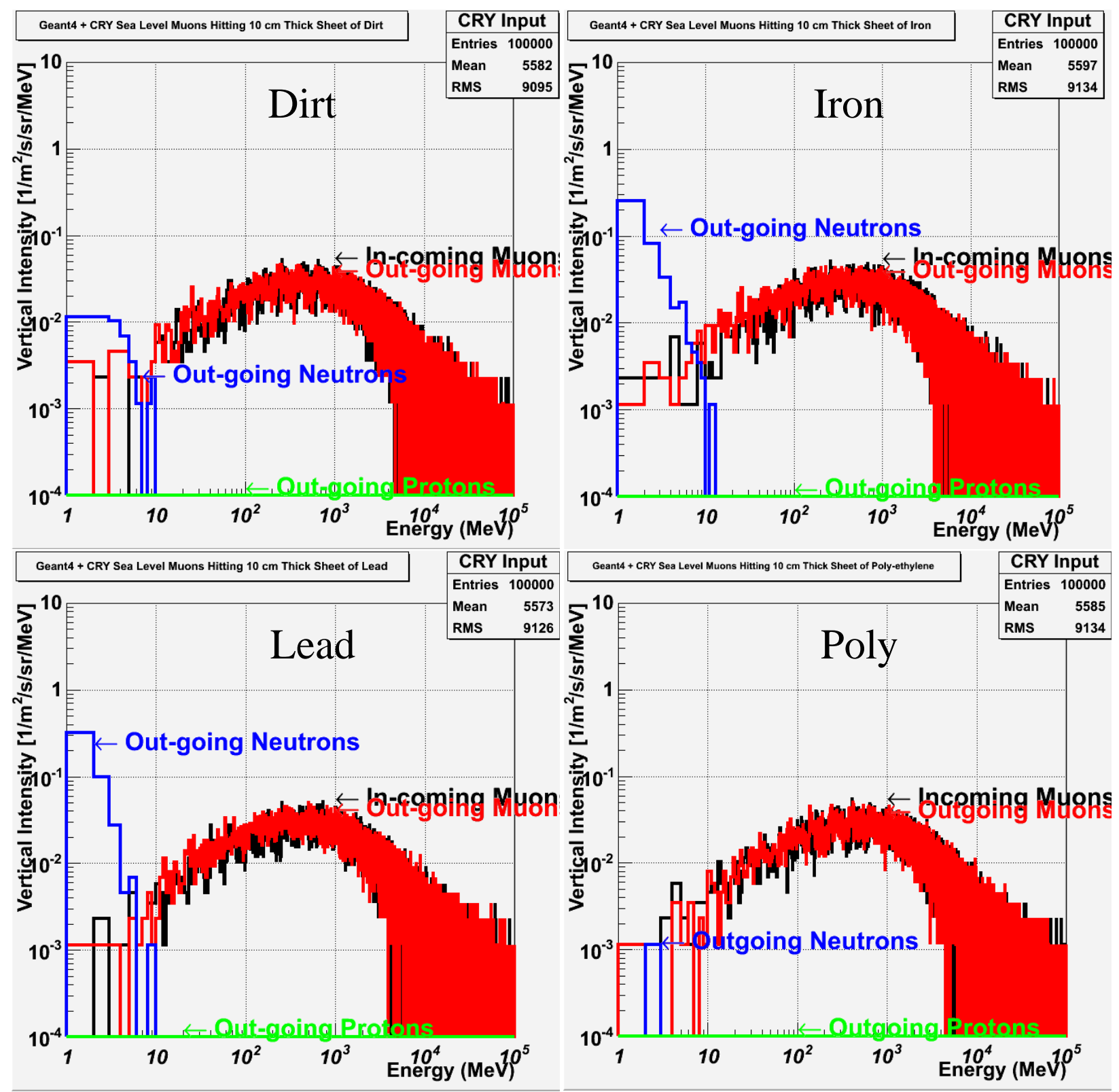

Figure 3: Simulation Results for Sea Level Muons Through $10 \mathrm{~cm}$ of Material. 


\subsection{Experimental validation of the application}

Simulated results require validation by comparison with experimental measurements. Cosmic rays have such a wide energy range and different particle components that is hard to find an experiment in which an apples to apples comparison can be made. Measurements of cosmic ray attenuation using the muon component of the cosmic shower are very frequently performed. The experiment uses two identical scintillator paddles vertically separated by an optimized distance and counts coincidences to tag muons. Usually the measurements take place at two locations and the ratio of muon fluxes can give an estimate of the equivalent depth. Such an experiment was carried out at PNNL to determine the attenuation factor of cosmic rays in the shallow underground laboratory (Building 3425) [Kouzes et al. 2009]. In this section the results of the MaCoR application is presented for the same environment as described in [Kouzes et al. 2009] and compared with experimental results. The geometry of the simulation is identical to that of the previous section. The difference here is that instead of changing the simulated material, the thickness of the material is changed. The simulated material is dirt. Figure 4 shows the experimental ratio of intensity normalized to intensity at sea level from MaCoR and [Kouzes et al. 2009]. There are analytical models for muon intensity vs. depth. Figure 4 also shows the results of the analytical approach by Barbouti and Rastin [Grieder 2001] to calculate attenuation versus depth. This model was developed for measurements at deep locations (>1000 ft.) and in this study we assume it is valid for shallow depths (0-50 ft.). All results fall within a 10\% deviation in dirt depths over $30 \mathrm{ft}$. All simulation results presented in this section are of runs of $10^{5}$ source events. The simulations were run several times with different random numbers to calculate the statistical error of these results. The experimental result and the simulated result are within a 3\% deviation. Figure 5 shows the muon spectra from the PNNL Shallow Lab simulation. It is worth noting the secondary neutron generation at this depth can reach up to $10 \mathrm{MeV}$.

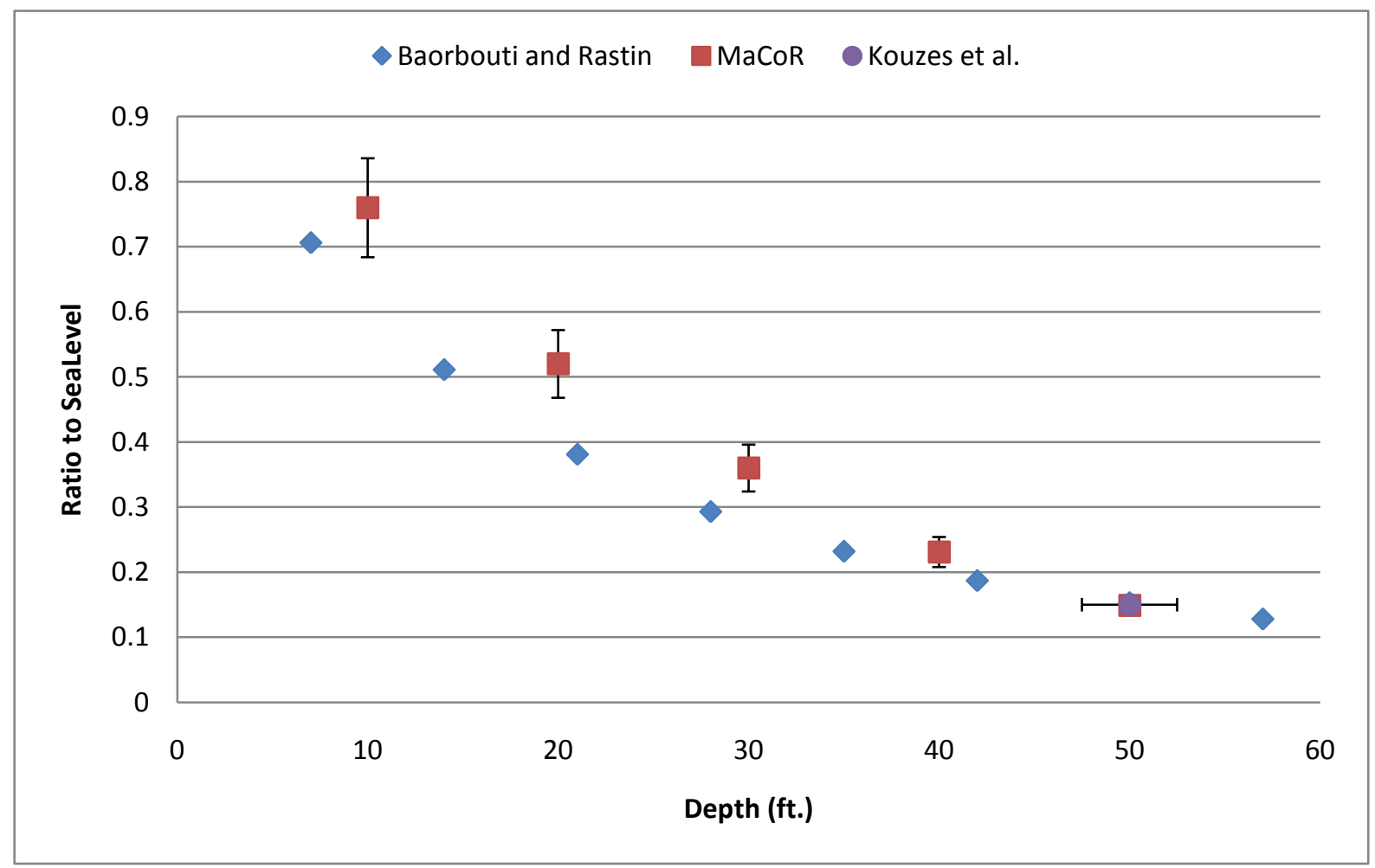

Figure 4: Muon Attenuation as a Function of Dirt Depth. 


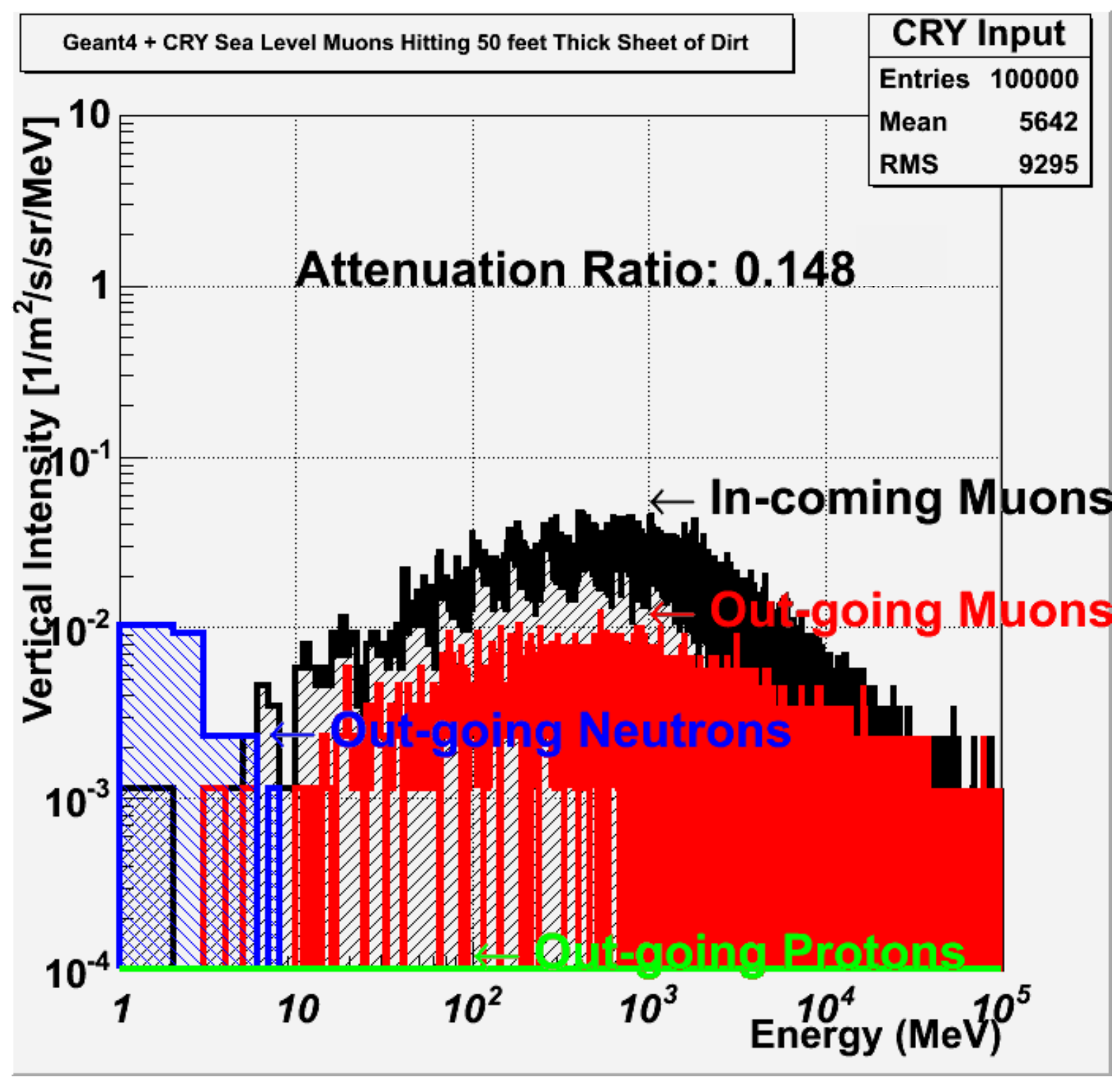

Figure 5: Simulation Result of the PNNL Shallow Lab.

The mean energy of the muon flux cannot be determined experimentally using the detector presented in [Kouzes et al. 2009]. The MaCoR application can give this information. Figure 6 shows the mean energy of the muons that survive the sheet of dirt versus depth. The trend denotes an increase in mean energy as a function of depth. The low energy muons are attenuated whereas the high energy ones have more penetrating power. At higher depths, the muon flux might be lower but the mean energy of these muons is much higher. Figures 7,8 and 9 show the different components of the cosmic shower at sea level and their attenuated differential flux when exposed to 10, 20, 30, 40 and 50 feet of dirt. 


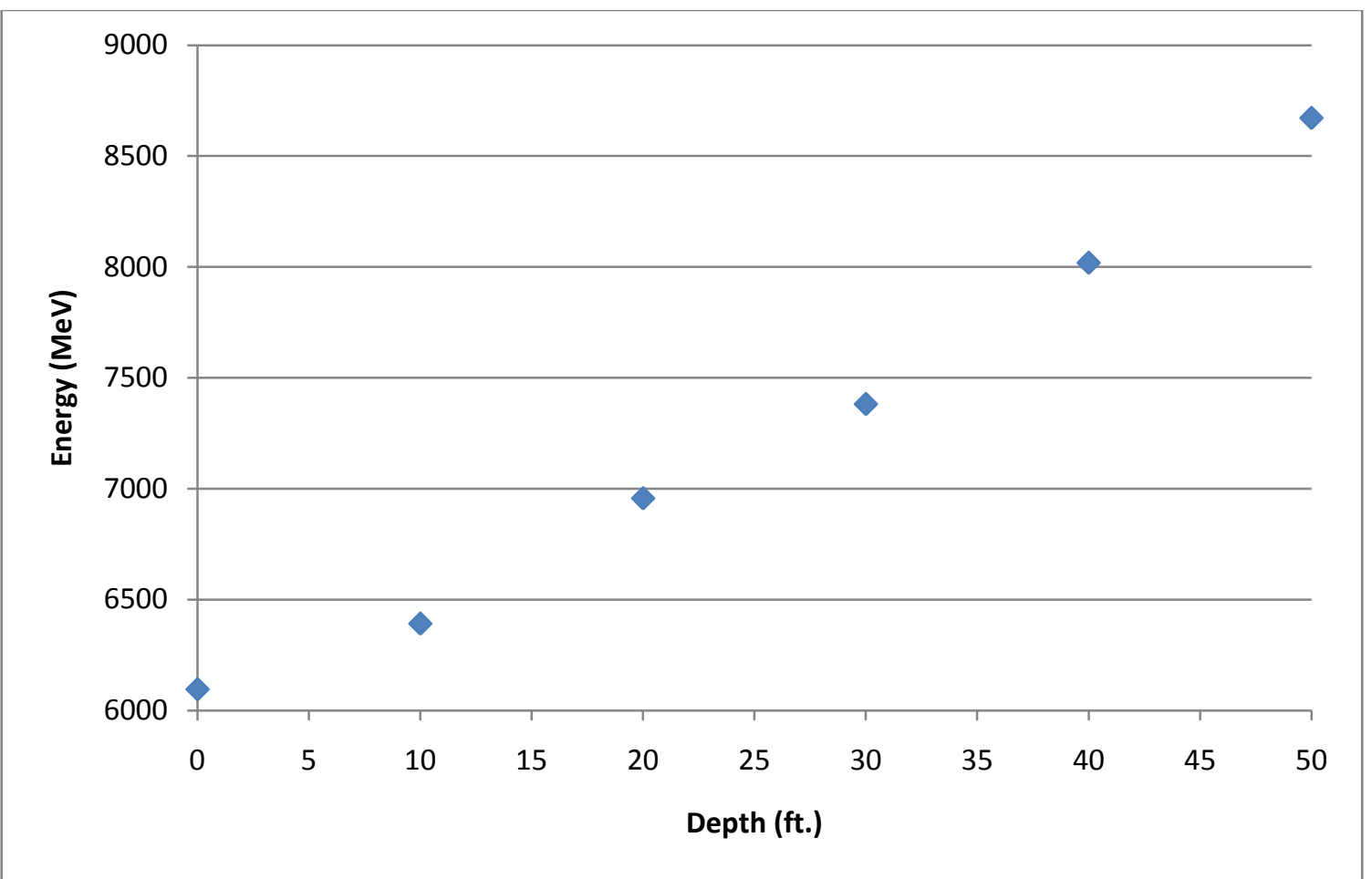

Figure 6: Simulated Muon Flux Mean Energy as a Function of Depth 


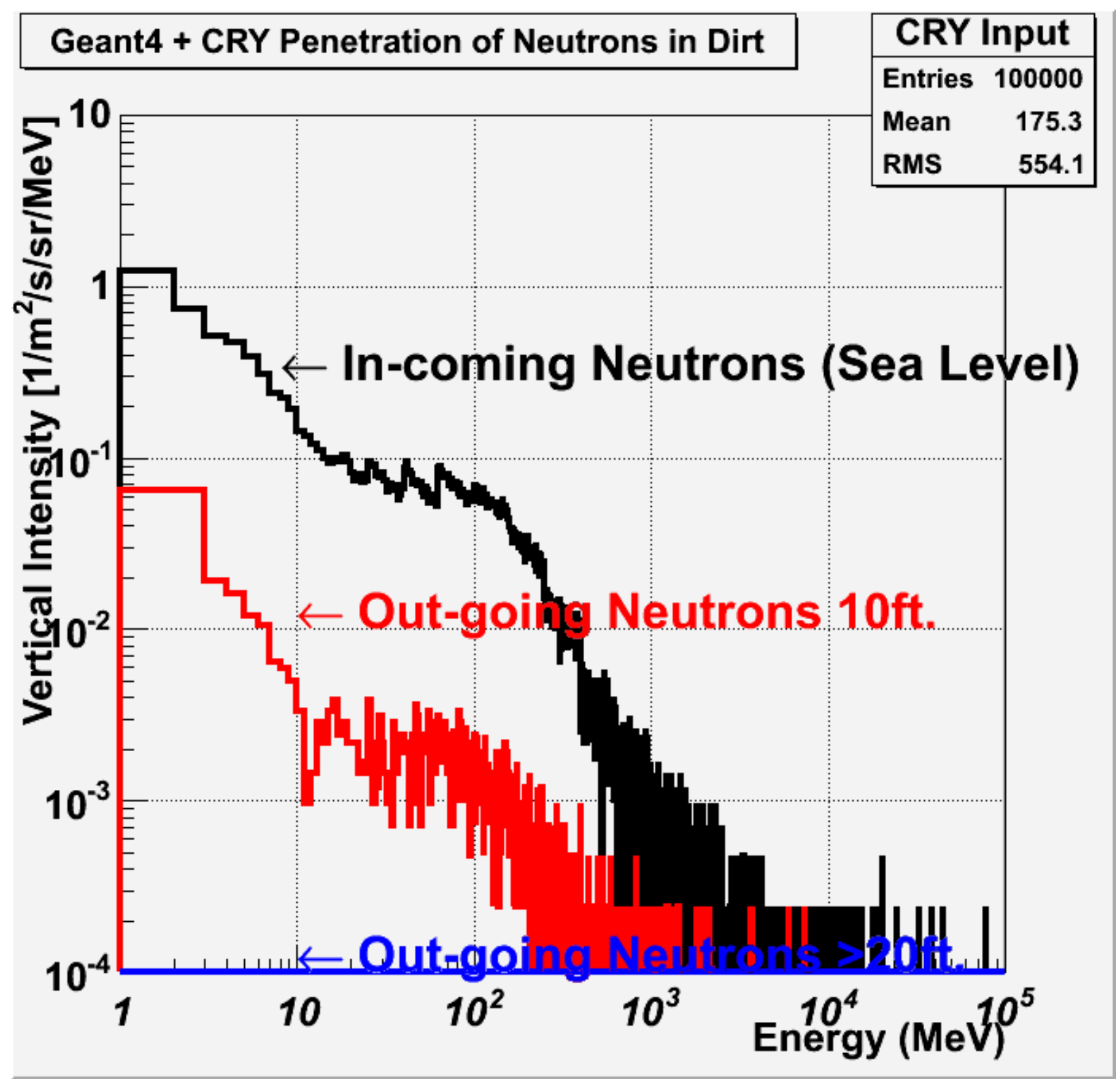

Figure 7: Neutron Penetration in Dirt 


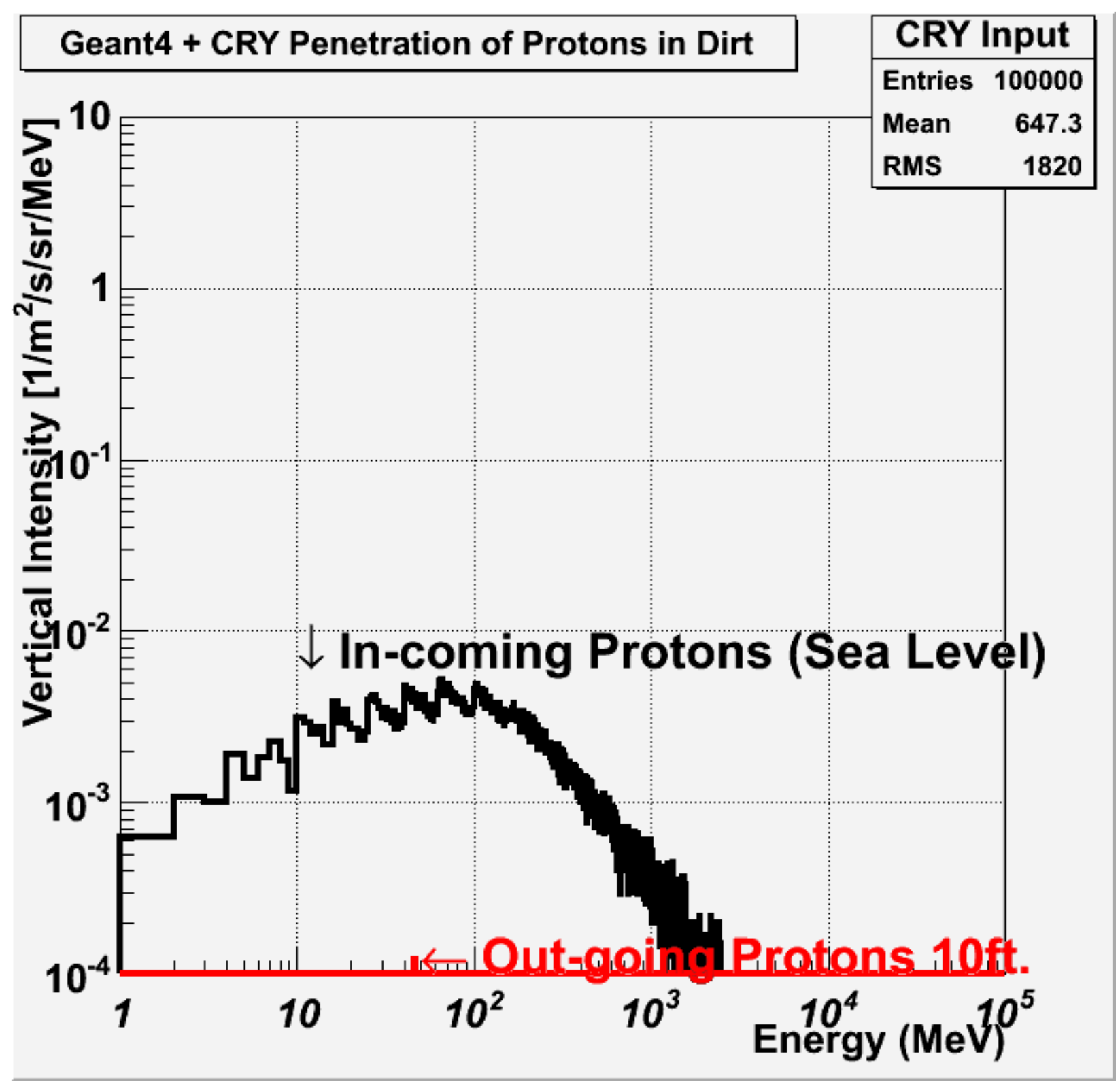

Figure 8: Proton Penetration in Dirt 


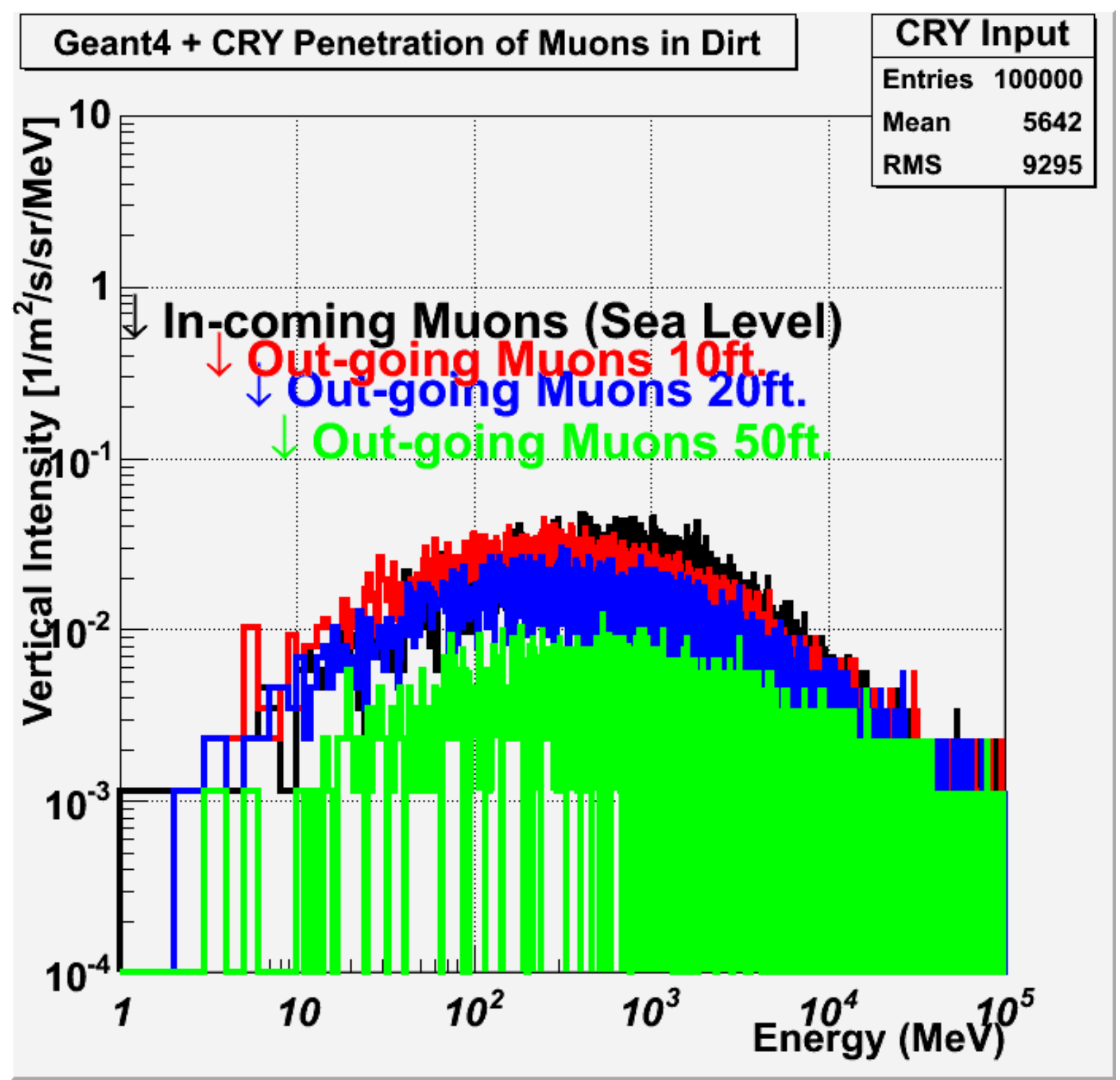

Figure 9: Muon Penetration in Dirt 


\subsection{Conclusions}

An application that simulates cosmic ray interactions in different media has been presented. The simulation tool has been developed using the hadronic and electromagnetic physics of the Geant 4 toolkit and the input of the CRY cosmic ray library. The tool is used to model the interaction of cosmic rays with four different materials of interest for shielding design (dirt, iron, lead and poly). The simulations were carried out separately for each particle component of interest for shielding applications (neutrons, protons and muons). The results show that generation of secondary neutrons by muons is implemented in Geant 4 ; whether this is muon capture generated neutrons or boil off neutrons from other muon interactions remains to be studied. The accuracy of muon attenuation predicted by the application is better than $4 \%$ when compared with experimental muon flux measurements in a shallow facility (depth of $50 \mathrm{ft}$ ). This simulation shows how the proton and neutron components of cosmic showers at sea level are completely eliminated with $20 \mathrm{ft}$. of dirt. Further studies examining shield designs for material transport and storage, are ongoing. 


\subsection{References}

[Aalseth 2010] Aalseth CE, E Aguayo, M Amman, FT Avignone III, HO Backe, X Bai, AS Barabash, PS Barbeau, M Bergevin, FE Bertrand, M Boswell, V Brudanin, W Bugg, TH Burritt, M Busch, G Capps, YD Chan, JI Collar, RJ Cooper, R Creswick, JA Detwiler, J Diaz, PJ Doe, Y Efremenko, V Egorov, H Ejiri, SR Elliott, J Ely, J Esterline, H. Farach, JE Fast, N Fields, P Finnerty , FM Fraenkle, VM Gehman, GK Giovanetti, M Green, VE Guiseppe, K Gusey, AL Hallin, GC Harper, R Hazama, R Henning, A Hime, H Hong, EW Hoppe, TW Hossbach, S Howard, MA Howe, RA Johnson, KJ Keeter, M Keillor, C Keller, JD Kephart, MF Kidd, A Knecht, O Kochetov, SI Konovalov, RT Kouzes, BH LaRoque, L Leviner, JC Loach, PN Luke, S MacMullin, M.G. Marino, R.D. Martin, D Medlin, D-M Mei, HS Miley, ML Miller, L Mizouni, AW Myers, M Nomachi, JL Orrell, D Peterson, DG Phillips II, AWP Poon, O Perevozchikov, G Perumpilly, G Prior, DC Radford, D Reid, K Rielage, RGH Robertson, L Rodriguez, MC Ronquest, H Salazar, AG Schubert, T Shima, M Shirchenko, V Sobolev, D Steele, J Strain , G Swift, K Thomas, V Timkin, W Tornow, TD Van Wechel, I Vanyushin, RL Varner, K Vetter, K Vorren, JF Wilkerson, BA Wolfe, W Xiang, E Yakushev, H Yaver, AR Young, C-H Yu, V Yumatov and C Zhang (The Majorana Collaboration). 2010a. "The MAJORANA Experiment." In Proceedings of the Neutrino Oscillation Workshop (NOW2010), September 4-11, 2010, Conca Specchiulla, Italy.

[Agostinelli 2003] Agostinelli, S “Geant4 - a simulation toolkit”, Nucl. Instr. And Meth. A 506, 2003 pp. $250-303$ (2003)

[Barabanov 2006] Barabanov I, Belougurov S, Bedrukov L, Denisov L, Kornoukov V, Sobolevsky N. 2006.

Cosmogenic Activation of germanium and its reduction for low background experiments. Nucl. Instr. And Meth. B B 251 pp 115-120.

[BIO 2011] Using biosolids for reclamation and remediation of disturbed soils Accessed March 12, 2011 at http://www.nps.gov/plants/restore/pubs/biosolids/why.htm.

[Bogdanov 2006] Bogdanov AG, "Geant4 simulation of production and interaction of muons", IEEE Trans. Nucl. Scie. 53513

[GEANT 2011] Accessed March 10, 2011 at http://geant4.cern.ch/support/proc_mod_catalog/models/hadronic/BertiniCascade.html.

[Grieder 2001] Grieder, PKF. “Cosmic Rays at Earth”. Elsevier, 2001.

[Hagmann 2011] Hagmann C, Lange D, Wright D “Cosmic-ray Shower Library (CRY)” LLNL UCRL-TM-229453

Lawrence Livermore National Laboratory. Avaliable at http://nuclear.llnl.gov/simulation/cry.pdf

[Kouzes et al. 2009] Kouzes RT, Borgardt JD, Lintereur AT, Panisko ME. 2009. Assessment of Cosmic Background Attenuation at Building 3425 PNNL-18855, Pacific Northwest National Laboratory, Richland, WA.

[NNDC 2011]Lawrence Livermore National Laboratory National Nuclear Data Center, Accessed March, 2520011 at http://www.nndc.bnl.gov

[Pia 2009] Pia, MG “Geant4 in Scientific Literature” Preprint at arXiv:0912.0360v1 (2009) 
[ROOT 2011] ROOT European Center for Nuclear research (CERN) Accessed April 1, 2011 at http://root.cern.ch/root/Reference

[Stewart 2006] Stewart DY, Harrison PF, Morgan B, Ramachers Y. 2006. Radiation shielding of underground low-background experiments. Nucl. Instr. And Meth. A 571 pp 651-662. 



\section{Distribution}

No. of

Copies

\# Name

Organization

Address

City, State and ZIP Code

\# Organization

Address

City, State and ZIP Code

Name

Name

Name

Name

Name (\#)

\# Name

Organization

Address

City, State and ZIP Code
No. of

\section{Copies}

\section{\# Foreign Distribution}

\# Name

Organization

Address

Address line 2

COUNTRY

\# Local Distribution

Pacific Northwest National Laboratory

Name

Name

Mailstop

Mailstop

Name

Mailstop

Name

Mailstop

Name

(PDF) 


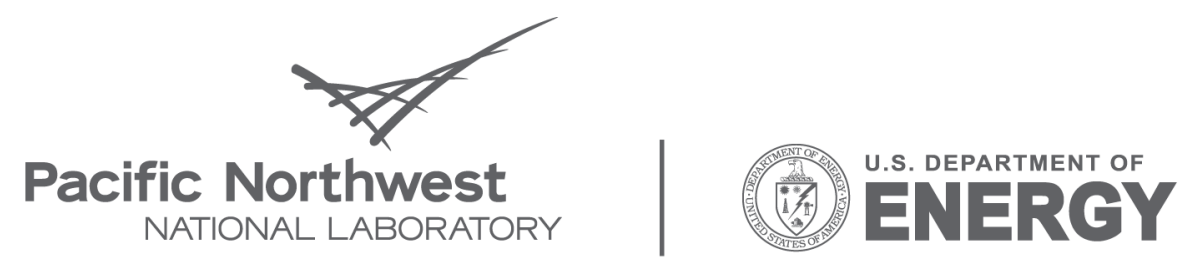

Proudly Operated by Battelle Since 1965

902 Battelle Boulevard

P.O. Box 999

Richland, WA 99352

1-888-375-PNNL (7665)

www.pnl.gov 